\title{
Differential Susceptibility of Human Cancer Cell Lines to Wild-Type Tanapoxvirus Infection
}

\author{
Hui Lin Lee and Karim Essani*
}

Laboratory of Virology, Department of Biological Sciences, Western Michigan University, Kalamazoo, MI 49008, USA

\begin{abstract}
Tanapoxvirus (TPV) is a member of the genus Yatapoxvirus in the family Poxviridae and is endemic to equatorial Africa. This disease is restricted to human and non-human primates, producing a mild febrile illness characterized by a single or more rarely additional pock-like lesions on the extremities. While there are several studies elucidating the replication cycle and host range of TPV, there is currently no standardized investigation comparing the ability of TPV to successfully replicate in a variety of tumor cell lines. This study examined the cytopathic effect and calculated the efficiency of TPV replication in vitro using 14 different human cancer cell lines. TPV replicates efficiently in some human tumor cells, and is restricted in others when measured by viral titer at 7 days post infection. Results described here clearly demonstrate that TPV replication in one glioblastoma cell line (U-373), and one colorectal cancer cell line (HCT-116) is more productive than in owl monkey kidney cells (OMK). Replication in two renal cancer cell lines (ACHN and Caki-1) is also increased when compared to OMK. TPV infection produced the greatest change in cellular morphology in U-373 cells, and to a much lesser degree in the breast cancer cell lines T-47D and MCF-7, and in the ovarian cancer line SK-OV3. Negligible change was noted in glioblastoma line U-87, breast cancer line MDA-MB-435, osteosarcoma line HOS, melanoma line SK-MEL5, colorectal cancer line COLO205, and prostate cancer line PC3. The cell lines least permissive to TPV replication were the glioblastoma (U-87) and melanoma (SK-MEL5) cell lines.
\end{abstract}

Keywords: Tanapoxvirus, poxvirus, yatapoxvirus, glioblastoma, cell culture.

\section{INTRODUCTION}

Tanapoxvirus (TPV) was first described as the causative agent in two consecutive outbreaks among the Wapakomo tribe in 1957 and 1962 in the Tana River Valley, Kenya [1]. The virus was isolated during the 1962 outbreak, which affected over 50 people, and investigations conducted in Liverpool confirmed that it was a previously uncharacterized poxvirus [1]. Both outbreaks coincided with severe and extended flooding of the Tana River, eliciting a hypothesis that TPV infection is a zoonotic disease transmitted by mosquitoes or other blood-feeding insect vectors. Later incidences of TPV cases in Zaire affecting riverine natives supported this hypothesis [2].

Patients infected with TPV sometimes suffered from a low-grade fever that lasted for 2-4 days [2]. The formation of one and rarely more pock-like skin lesions occurred after this short febrile illness. In the majority of cases, the development of a $1.0 \mathrm{~cm}$ in diameter papule on the extremities was raised, necrotic and contained no liquid unlike the pustular lesions of monkeypox [1,2]. Regional lymphadenopathy also developed by the end of the first week of infection. In the following week, the papule ulcerated but never spread to more than $2.0 \mathrm{~cm}$ in diameter. The infection usually resolved within 6 weeks, when the ulcer heals and a scar forms and disappears at the site of ulceration, with little discomfort reported by patients.

*Address correspondence to this author at the Department of Biological Sciences, Western Michigan University, 3441 Wood Hall, Kalamazoo, MI 49008, USA; Tel: (269) 387-2661; Fax: (269) 387-5609;

E-mail: karim.essani@wmich.edu
TPV is classified in the family Poxviridae, and is a member of the genus Yatapoxvirus [3]. There are two species in this genus officially recognized by the International Committee for Taxonomy of Viruses (ICTV), TPV, and Yaba monkey tumor virus (YMTV) [4]. Yaba-like disease virus (YLDV) is over 98\% similar in terms of genomic homology to TPV and is considered a strain of TPV $[5,6]$. Outbreaks of what is now termed YLDV [7] occurred in several primate facilities in the United States in 1965 and 1966 when an initial infection that affected several monkey species spread to their human handlers [8-10]. Subsequent investigations revealed that YLDV and TPV have the same host range and produced the same lesions in susceptible animals [8].

Morphologically, TPV measures between 250 and 300 $\mathrm{nm}$ in size and is similar to the prototypical member of the orthopoxvirus genus, vaccinia virus (VV) [11]. These large brick-shaped virions come in two distinct infectious forms, the intracellular mature virion (IMV) and extracellular enveloped virion (EEV). The majority of virions exist in the enveloped form [3]. In vitro, the replication cycle of TPV ranges from 36 to 48 hours depending upon the multiplicity of infection [12]. This is significantly slower than the 24 hour replication cycle of VV [13]. TPV contains 144,565 base pairs in its linear double-stranded DNA genome that was fully sequenced in 2007 [14]. TPV carries out DNA replication in specific areas of the cytoplasm of the host cell, assembling its new IMV particles in viral factories. The DNA genome of poxviruses contains inverted terminal repetitions (ITR), and is connected by hairpin loops [11]. The central region of the poxvirus genome is highly conserved for structural and essential replication genes. The 
termini tend to contain virulence and host-range genes that may be species-specific [5]. The host ranges of YLDV and TPV have been evaluated in a variety of cell lines of animal origin, as well as in live animals. Neither TPV nor YLDV replicate in chick embryo fibroblasts, primary and continuous rabbit kidney cells, bovine kidney cells, nor do they cause any lesions in mice, hamsters, and guinea pigs [ $[1$, $8,10]$. There has been one report that YLDV caused lesions on German checker rabbits, but it does not cause the same effect in New Zealand white rabbits [10]. YLDV does not cause any cytopathic effect in adult rabbit cell lines [8]. Nevertheless, TPV has been shown to replicate in rat lung cells [12]. Currently, there are no studies that evaluate the susceptibility of different human cancer cell lines to TPV in a standardized protocol. TPV has been cultured in several human cell lines such as primary human dermal fibroblasts, primary human peripheral blood mononuclear cells, primary human thyroid, amnion, and human fetal lung cells, and several human cancer cells such as human epidermoid carcinoma and cervical cancer $[1,8,12,15]$. The long-term objective of these studies is to evaluate the oncolytic potential of this virus in an animal model employing nude mice. Here we report the differential susceptibility of 14 different human cancer cell lines to TPV infection.

\section{MATERIALS AND METHODOLOGY}

\section{Virus, Cell Culture and Reagents}

TPV expressing the enhanced green fluorescent (eGFP) gene was a gift from Dr. Grant McFadden at the University of Florida, Gainesville, Florida. The eGFP was inserted between ORF 5L and ORF 6L of TPV-Kenya and is regulated by a synthetic early/late promoter as described by Nazarian et al. [15]. Owl monkey kidney (OMK), human glioblastoma (U-87, U-373, U-118), and human breast cancer (T-47D) cells were obtained from the American Type Culture Collection, Rockville, Maryland. Human osteosarcoma (HOS), human renal cell cancer (Caki-1, $\mathrm{ACHN}$ ), human ovarian cancer (SK-OV3), human breast cancer (MCF-7, MDA-MB 435), human prostate cancer (PC3), human colorectal cancer (COLO205, HCT-116), and human melanoma (SK-MEL5) cell lines were also a gift from Dr. Grant McFadden. OMK cells were cultured in Eagle's minimum essential medium (EMEM) purchased from Sigma, (St. Louis, MO), and supplemented with $10 \%$ (v/v) newborn calf serum (NBCS) from Life Technologies, (Grand Island, NY), 1.5g/L sodium bicarbonate, $2 \mathrm{mM} \mathrm{L-}$ glutamine, and antibiotics (penicillin G sodium 100 units $/ \mathrm{ml}$, and streptomycin sulfate $100 \mu \mathrm{g} / \mathrm{ml}$ ) from Invitrogen (Carlsbad, CA) (growth medium). Infected cell monolayers were maintained in EMEM supplemented with $2 \%(\mathrm{v} / \mathrm{v})$ NBCS, $1.5 \mathrm{~g} / \mathrm{L}$ sodium bicarbonate, L-glutamine, and antibiotics (maintenance medium). U-87, U-373, U-118, and T-47D cells were cultured in EMEM supplemented with $10 \%(\mathrm{v} / \mathrm{v})$ fetal bovine serum (FBS) purchased from Life Technologies, (Grand Island, NY), $1.5 \mathrm{~g} / \mathrm{L}$ sodium bicarbonate, $2 \mathrm{mM}$ L-glutamine, and antibiotics (penicillin G sodium 100 units $/ \mathrm{ml}$, and streptomycin sulfate $100 \mu \mathrm{g} / \mathrm{ml})$ purchased from Invitrogen (Carlsbad, CA) (growth medium). Infected cell monolayers were maintained in EMEM supplemented with $2 \% \quad(\mathrm{v} / \mathrm{v}) \quad \mathrm{FBS}, \quad 1.5 \mathrm{~g} / \mathrm{L}$ sodium bicarbonate, L-glutamine, and antibiotics (maintenance medium). HOS, MCF-7, COLO205, HCT-116, ACHN, SK-
MEL5, MDA-MB 435, and Caki-1 cells were cultured in Dulbecco's (modified Eagle medium DMEM) (Gibco) supplemented with $10 \%(\mathrm{v} / \mathrm{v}) \quad \mathrm{FBS}, \quad 1.5 \mathrm{~g} / \mathrm{L}$ sodium bicarbonate, $2 \mathrm{mM} \mathrm{L-glutamine,} \mathrm{and} \mathrm{antibiotics} \mathrm{(growth}$ medium). Infected cell monolayers were maintained in DMEM supplemented with $2 \%(\mathrm{v} / \mathrm{v})$ FBS, $1.5 \mathrm{~g} / \mathrm{L}$ sodium bicarbonate, L-glutamine, and antibiotics (maintenance medium). SK-OV3 was cultured in McCoy's 5a medium (ATCC) supplemented with $10 \%$ (v/v) FBS, $2.2 \mathrm{~g} / \mathrm{L}$ sodium bicarbonate, $2 \mathrm{mM} \mathrm{L-glutamine,} \mathrm{and} \mathrm{antibiotics} \mathrm{(growth}$ medium). Infected cell monolayers were maintained in McCoy's 5a medium supplemented with $2 \%(\mathrm{v} / \mathrm{v})$ FBS, $1.5 \mathrm{~g} / \mathrm{L}$ sodium bicarbonate, L-glutamine, and antibiotics (maintenance medium). PC3 was cultured in F-12K medium (ATCC) supplemented with $10 \%(\mathrm{v} / \mathrm{v}) \mathrm{FBS}, 1.5 \mathrm{~g} / \mathrm{L}$ sodium bicarbonate, $2 \mathrm{mM} \mathrm{L-glutamine,} \mathrm{and} \mathrm{antibiotics} \mathrm{(growth}$ medium). Infected cell monolayers were maintained in F$12 \mathrm{~K}$ medium supplemented with $2 \%(\mathrm{v} / \mathrm{v})$ FBS, $1.5 \mathrm{~g} / \mathrm{L}$ sodium bicarbonate, L-glutamine, and antibiotics (maintenance medium). All cell lines were incubated at $37^{\circ} \mathrm{C}$ in a humidified atmosphere of $95 \%$ air and $5 \% \mathrm{CO}_{2}$ unless otherwise noted.

\section{Virus Quantitation}

Approximately $1 \times 10^{5} \mathrm{TPV}$ virions expressing eGFP (TPV-GFP) in $600 \mu \mathrm{L}$ maintenance medium were inoculated into $25 \mathrm{~cm}^{2}$ monolayers of each cancer cell line. The virus was allowed to adsorb for 1 hour at room temperature on a rocking platform before non-adherent virus in the maintenance medium was aspirated out. Fresh maintenance medium was then added and each flask was incubated at $37^{\circ} \mathrm{C}$ for 7 days. Infected monolayers were subjected to three cycles of freeze-thawing to release all viral particles into the medium. This infected medium was then serially diluted and titrated in duplicates, onto confluent monolayers of OMK cells cultivated in 6-well dishes, using $200 \mu \mathrm{L}$ dilutions of 1 to $10^{-6}$. The virus was allowed to adsorb for 1 hour at room temperature before the addition of overlay medium (maintenance medium containing $0.5 \%(\mathrm{w} / \mathrm{v})$ methylcellulose 4000 centipoises (Fisher Scientific, NJ) and incubated at $37^{\circ} \mathrm{C}$ for 10-15 days. Once viral plaques were visible under the microscope, the medium was then removed from the wells and the monolayer was stained with $0.1 \%(\mathrm{w} / \mathrm{v})$ crystal violet in $10 \%$ formaldehyde (plaque staining solution). The monolayer stained for 20 minutes, rinsed in deionized water and air-dried. The average number of plaques was counted to determine the total yield of virus recovered from the infected flask.

\section{Visualization of Cells}

At 7 days post infection, infected and uninfected cells in well plates were visualized using a Nikon Diaphot 300 epifluorescence microscope under bright light or through a B2A filter cube (450 to $490 \mathrm{~nm}$ excitation band and 520 rim barrier filter). All photographs were taken using a $35 \mathrm{~mm}$ camera and processed through the Metamorph Image Analysis software from Molecular Devices (Downingtown, PA).

\section{RESULTS}

The ability of TPV-GFP to replicate in and destroy various human cancers was examined in fourteen human 
cancer cell lines. TPV-GFP was inoculated into breast cancer cells (T47D, MCF-7, MDA-MB-435), glioblastoma cells (U87, U-373, U-118), osteosarcoma cells (HOS), melanoma cells (SK-MEL5), ovarian cancer cells (SK-OV-3), renal cancer cells (ACHN, Caki-1), colorectal cancer cells (COLO205, HCT-116), and prostate cancer cells (PC3). OMK cells were used as a control. Complete viral titers are listed in Table 1. The images in Fig. (1) show TPV-GFP infected control cells (OMK) and cancer cells under visible and ultra violet light at 7 days post infection (dpi).

Table 1. Titer of TPV-GFP from Infected Cancer Cell Lines at 7 Days Post Infection

\begin{tabular}{|c|c|c|}
\hline \multicolumn{2}{|l|}{ Cell Lines } & \multirow{2}{*}{$\begin{array}{c}\begin{array}{c}\text { Total Virus } \\
\text { Yield in } 5 \mathbf{~ m}\end{array} \\
1.0125 \times 10^{6}\end{array}$} \\
\hline Control (Owl Monkey Kidney) cells & OMK & \\
\hline \multirow{3}{*}{ Human Breast Cancer Cells } & T47D & $7.25 \times 10^{4}$ \\
\hline & MCF-7 & $1.7375 \times 10^{5}$ \\
\hline & MDA-MB-435 & $5.25 \times 10^{5}$ \\
\hline \multirow{3}{*}{ Human Glioblastoma Cells } & $\mathrm{U}-118$ & $2.875 \times 10^{5}$ \\
\hline & U-373 & $2.25 \times 10^{7}$ \\
\hline & $\mathrm{U}-87$ & $4.375 \times 10^{3}$ \\
\hline Human Osteosarcoma Cells & HOS & $5.5 \times 10^{4}$ \\
\hline Human Melanoma Cells & SK-MEL5 & $4.25 \times 10^{3}$ \\
\hline Human Ovarian Cancer Cells & SK-OV-3 & $1.9625 \times 10^{5}$ \\
\hline \multirow{2}{*}{ Human Renal Cancer Cells } & $\mathrm{ACHN}$ & $7.75 \times 10^{6}$ \\
\hline & Caki-1 & $4.0 \times 10^{6}$ \\
\hline \multirow{2}{*}{ Human Colorectal Cancer Cells } & COLO205 & $4.5 \times 10^{4}$ \\
\hline & HCT-116 & $1.05 \times 10^{7}$ \\
\hline Human Prostate Cancer Cells & $\mathrm{PC} 3$ & $6.5 \times 10^{4}$ \\
\hline
\end{tabular}

Cells were grown in $25 \mathrm{~cm}^{2}$ flasks to confluency and each flask was infected with approximately $1 \times 10^{5} \mathrm{pfu}$. Infected flasks were incubated at $37^{\circ} \mathrm{C}$ for 7 days, and total virus yield was quantitated as described in Materials and Methods.

TPV-GFP replicated poorly in T47D and MCF-7 cells compared to OMK cells. There was a 14-fold and 6-fold reduction in number of virus particles retrieved from T47D and MCF-7 respectively, while MDA-MB-435 produced a 2fold reduction in viral particles when compared to OMK cells. Green fluorescence observed in T47D and MCF-7 is predictably less than in OMK cells. Cytopathic effect (CPE) in T47D and MCF-7 was less pronounced than CPE observed in OMK cells. There was no eventual destruction of cells, and T47D and MCF-7 monolayers remained intact even after two weeks. There was no identifiable CPE observed in virus infected MDA-MB-435 monolayer.

TPV-GFP replicated poorly in U-118 and U-87 when compared to OMK cells, with a 3.5 -fold reduction in U-118 cells, and a dramatic 231-fold reduction in viral replication in U-87 cells. In contrast, replication in U-373 surpassed replication in OMK by 22 -fold. Replication in U-373 cells was the most productive among all 14 cell lines tested. CPE was observed in U-118 and U-373 cells, but not at all discernable in U-87 cells. Green fluorescence was noted to also be present within U-87 neurospheres despite the lack of visible CPE. TPV infected U-373 cells exhibited the most pronounced CPE where entire infected cell monolayers detached completely from the surface of the flask.

TPV-GFP produced 18-fold less virus particles in osteosarcoma cells (HOS) compared to OMK. There was observable CPE in the HOS infected monolayer, but no plaques were formed at 7 days. No further progression of CPE was noted beyond the initial infected area seen in Fig. (1).

In melanoma (SK-MEL5) cells, there was a 238-fold decrease in average number of viral particles recovered. There was no truly distinct CPE observed in infected SKMEL5 cells, and no plaques formed. The cell monolayer also remained intact well beyond 7 days post inoculation. SKMEL5 cells were the least suitable host for TPV-GFP replication among all cell lines tested.

TPV-GFP in ovarian cancer cells (SK-OV-3) suffered a 5 -fold reduction compared to that obtained from OMK cells. Infected SK-OV-3 cells appeared to become larger, flatter in appearance, more granular, and appeared darker in color, than uninfected SK-OV-3 cells. TPV-GFP yielded higher titers in the renal cancer cell lines tested compared to OMK cells at 7 days post infection.

The average viral replication of TPV-GFP in ACHN cells was more than 7 -fold greater than OMK cells, while the average replication in Caki-1 cells was nearly 4-fold greater than in OMK cells. There was a drastic change in cellular morphology of virus infected ACHN and Caki-1 monolayers. When the uninfected ACHN monolayer was fully confluent, infected ACHN monolayers had prominent CPE. The infected ACHN monolayer developed bright green foci under UV light, while areas of destroyed cells were seen as large clearings. Infected cells also become stretched, similar to that seen in a later stage of infected OMK cells. On the other hand, infected Caki-1 cells became more spherical and spread out, from the original spindle-like shape of tightly packed uninfected confluent monolayers. An increase in cellular debris in infected Caki-1 cell medium also indicated that cell death occurred, causing detachment of cells from the surface of the flask, and subsequently reducing the number of cells observed.

There was an interesting difference between two lines of human colorectal cells examined. While the average replication of TPV-GFP in COLO205 cells was close to 23fold lower than in OMK cells, replication in HCT-116 cells was more than 10-fold greater compared to OMK cells. COLO205 cells are semi-adherent in cell culture, and have varied cell morphology. The TPV-GFP infected COLO205 cells did not exhibit a noticeable change in cell morphology under visible light. HCT-116 cells, on the other hand, did show significant $\mathrm{CPE}$ at 7 days post inoculation. Viral plaques were clearly seen in infected HCT-116 monolayers, and some cells do develop the stretched cell morphology seen in OMK and ACHN cells.

TPV-GFP replication in prostate cancer (PC3) cells was almost 16-fold less than replication in OMK cells at 7 days post infection. There was no discernable CPE observed in virus infected PC3 monolayers, nor was there any apparent 


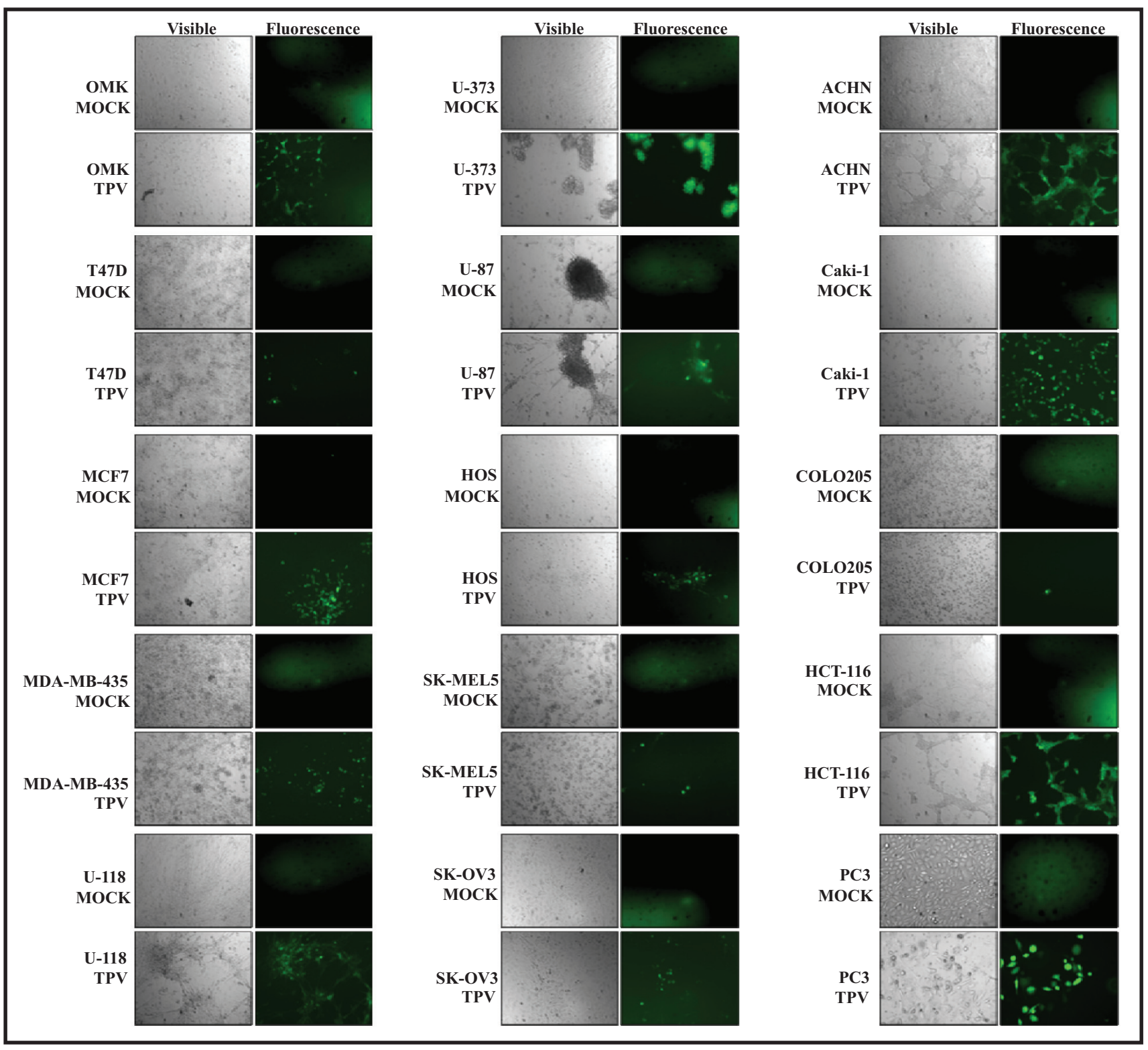

Fig. (1). UV fluorescent microscopy of TPV-GFP infected cancer cells at 7 days post infection. Left panels in each case show cell monolayers under visible light. Right panels show same area under UV fluorescence. Uninfected cells (MOCK) show no green fluorescence while TPV-GFP infected plaques emit green fluorescence. Greater progression of TPV-GFP infection is observed in U-373, HCT-116, ACHN, and Caki-1cell monolayers when compared to the OMK control.

change in cellular morphology. There was also no progression of green fluorescence beyond those initially recorded at day 7 , suggesting that viral replication was aborted in PC3 cells.

\section{DISCUSSION}

The ultimate aim of this study is to develop an oncolytic TPV that will be able to replicate in a variety of human cancer cells and drastically less or none at all in normal human cells. The results presented here demonstrate that TPV-GFP replicated well in several human cancer cell lines. Cytopathic effect was clearly observed in glioblastoma cell lines U-373 and U-118, renal cancer lines ACHN and Caki1, and colorectal cancer line HCT-116. Virus replication proceeded much faster in human cancer cells and also virus infection spread more quickly when compared to virus infection in OMK cells. Infected U-373 monolayers were completely destroyed and all infected cells detached from the surface of the culture flask within 7 days. There was a change in cellular morphology in breast cancer cell lines T47D and MCF-7, and to a lesser extent in the ovarian cancer line SK-OV3. Lastly, negligible morphological change was noted in glioblastoma line U-87, breast cancer line MDAMB-435, osteosarcoma line HOS, melanoma line SK-MEL5, colorectal cancer line COLO205, and prostate cancer line PC3. In these studies expression of GFP and/or CPE does not absolutely correlate with the production of mature virus. 
However, the titration of infectious virions does indicate the degree of virus productivity.

Initial green fluorescence observed in all infected human cancer cell lines tested indicates that TPV successfully penetrates all types of cells. Poxviruses enter the host cell through ubiquitous surface receptors like heparan sulfate, chondroitin sulfate and glycosaminoglycans, and this could explain the penetration of TPV and subsequent expression of early proteins including the green fluorescent protein $[16$, 17]. Poxviruses are equipped with their own replication machinery for mRNA synthesis and DNA replication, and since the GFP gene is regulated by a synthetic early/late promoter, it will express GFP at all stages of replication. However, TPV is not able to produce as many infectious progeny in several cell lines as efficiently as that seen in OMK cells. These results suggest that TPV-GFP replication in cell lines such as SK-MEL5, T-47D, and HOS could be abortive. This could be due to required factors that are missing or factors already present that abort the replication of TPV at later steps of the virus replication cycle. One possibility could be caused by an inability of infectious mature virus to exit the host cell to spread to nearby cells. The question that the degree of virus replication in a specific cell line is related to a specific stage of virus replication, like virus entry or virus uncoating remains open.

Despite the many similarities in gene homology and genomic organization between TPV and VV, the proteins in TPV are more closely related to myxoma virus than to VV [18] as determined from the fully sequenced genome of TPV and myxoma virus [19]. Myxoma virus is from the genus Leporipoxvirus and is host-restricted to rabbits, causing the highly lethal myxomatosis disease in European rabbits [3]. This virus has become of significant interest to humans as recent studies have shown that myxoma virus displays oncolytic properties in human cancers. This predilection towards cancer cells is attributed to the innate dysregulated intracellular signaling pathway of these abnormal cells [20]; specifically, the amplification and overexpression of a particular group of serine/threonine protein kinases belonging to the protein kinase $\mathrm{B}$ (PKB) family [21]. Three of these protein kinases AKT1, AKT2, and AKT3 within the AKT family, are highly conserved cellular homologues that are involved in a multitude of cellular growth functions such as inhibition of apoptosis [22], proliferation, cell metabolism, and possibly angiogenesis [23]. Constitutively activated/hyperactivated AKT is involved in oncogenesis [24] as cells cease to enter the quiescent phase of the cell cycle, resulting in non-stop cell proliferation.

One of the myxoma host range proteins, M-T5, binds to and activates Akt [25], which subsequently inhibits the mammalian Target of Rapamycin (mTOR) complex [26], allowing the virus to replicate within the cell. Similar to TPV host range proteins, M-T5 also contains ankyrin repeats. Myxoma virus has been shown to replicate in myriad glioblastoma cell lines that possess a higher basal level of endogenous phosphorylated Akt [26-29]. Besides glioblastoma cells, human pancreatic adenocarcinoma cell lines with this same abnormality were also found to be permissive to myxoma virus infection [30]. Non-permissive tumor cells can be modified to become susceptible to myxoma infection simply by activating Akt expression [31].
TPV is armed with a variety of potential immunomodulatory proteins such as Tumor Necrosis Factor binding, chemokine inhibitors, apoptosis inhibitors, complement inhibition, and other useful mechanisms to avoid and attack the host response to infection [14]. Nazarian et al. (2007) provided evidence that TPV also has many proteins showing ankyrin repeats and a 19L Kelch protein. Ankyrin repeats, like Kelch motifs mediate protein-protein interactions and suggests the same role for these uncharacterized proteins in TPV [32].

However, results from myxoma virus [31] do not correlate with results described here for TPV in terms of cell permissiveness to virus due to endogenous Akt. TPV-GFP replicates poorly in HOS cells that contain high endogenous and phosphorylated Akt, whereas myxoma virus replicates well in this osteosarcoma cell line [31]. Another difference is the MDA-MB-435 breast cancer cells with no detectable levels of phosphorylated Akt, yet is still permissive to TPVGFP replication [31].

It would be interesting to examine the susceptibility of human tumors to TPV in vivo since poxviruses are limited by their virulence genes, which are not always essential for replication in cell culture, but necessary to produce disease in an animal. Even though TPV is able to replicate in African green monkey kidney cell lines, it cannot cause an infection in African green monkeys $[8,33]$. These experiments would give us great insight on the in vivo host range of TPV.

Several poxviruses, (e.g. YLDV, VV and myxoma virus) have been evaluated for their ability to replicate in cancerous cells of human origin. Results from these studies indicate that these cancer killing viruses, or oncolytic viruses, are able to preferentially target these aberrant cells, suggesting that TPV could also possess anti-cancer abilities [30,34]. VV has progressed the furthest, of all the oncolytic poxviruses, and is currently undergoing clinical trials.

TPV would be a good candidate to be developed into an oncolytic virus because it is serologically distinct from VV and variola virus the causative agent of smallpox [1]. Thus, individuals vaccinated against smallpox with VV can still be treated with oncolytic TPV since it will not be subjected to immune clearance by the vast majority of the human population by VV specific antibodies.

We have previously shown that TPV replication in OMK cells is comparable to that in normal human fetal lung cells, WI-38 [12]. The results obtained from the current study provide a good foundation for subsequent experiments to illuminate the reasons for the increase in viral titer in certain cancer cell lines when compared to OMK cells. We are now in the process of inducing human xenograft tumors in nude mice to duplicate results presented here. In such studies the virus does not have to cross the blood brain barrier. However, wild type TPV is known to replicate in monocytes and macrophages that are capable of crossing the blood brain barrier.

\section{ACKNOWLEDGEMENTS}

We would like to acknowledge Dr. Robert Eversole for the use of the Biological Imaging Center at WMU. Dr. Bruce Bejcek and Dr. Grant McFadden kindly provided human cancer cell lines. We thank Steve Conrad for critically reviewing this 
manuscript. This study is a part of HL's MS thesis in this department, and was supported by a WMU grant to KE.

\section{REFERENCES}

[1] Downie AW, Taylor-Robinson CH, Caunt AE, et al. Tanapox: a new disease caused by a pox virus. Br Med J 1971; 1: 363-8.

[2] Jezek Z, Arita I, Szczeniowski M. Human tanapox in Zaire: clinical and epidemiological observations on cases confirmed by laboratory studies. Bull World Health Organ 1985; 63: 1027-35.

[3] Fenner F. Poxviruses. In: Fields, BN, Knipe, DM, Howley PM, et al. Eds. Fields Virology, $3^{\text {rd }}$ ed. Philadelphia: Lippincott-Raven Publishers 1996; pp. 2673-702.

[4] Fauquet C. Virus taxonomy: classification and nomenclature of viruses: eighth report of the International Committee on the Taxonomy of Viruses. San Diego, California: Elsevier Academic Press 2005.

[5] Brunetti CR, Amano H, Ueda Y, et al. Complete genomic sequence and comparative analysis of the tumorigenic poxvirus Yaba monkey tumor virus. J Virol 2003; 77: 3335-47.

[6] Knight JC, Novembre FJ, Brown DR, Goldsmith CS, Esposito JJ. Studies on Tanapox virus. Virology 1989; 172: 116-24.

[7] Hull RN. In: Gard S, Hallauer C, Meyer KF, Eds. The simian viruses. Virology Monographs, vol. 2. New York: Springer-Verlag 1968; pp. 25-30.

[8] España, C. A pox disease of monkeys transmissible to man. $2^{\text {nd }}$ Conference of experimental medicine and surgery in primates. Basel: Karger 1971; pp. 694-708.

[9] Hall AS, McNulty WP Jr. A contagious pox disease in monkeys. J Am Vet Med Assoc 1967; 151: 383-8.

[10] Crandell RA, Casey HW, Brumlow WB. Studies of a newly recognized poxvirus of monkeys. J Infect Dis 1969; 119: 80-8.

[11] Moss B. In: Fields BN, Knipe DM, Howley PM, et al., Eds. Fields Virology. Poxviridae: the viruses and their replication. Philadelphia: Lippincott-Raven Publishers 1996; 2637-2671.

[12] Mediratta S, Essani K. The replication cycle of tanapox virus in owl monkey kidney cells. Can J Microbiol 1999; 45: 92-6.

[13] Joklik W. The poxviruses. Bacteriol Rev 1966; 30: 33-66.

[14] Nazarian SH, Barrett JW, Frace AM, et al. Comparative genetic analysis of genomic DNA sequences of two human isolates of Tanapox virus. Virus Res 2007; 129: 11-25.

[15] Nazarian SH, Barrett JW, Stanford MM, et al. Tropism of Tanapox virus infection in primary human cells. Virology 2007; 368: 32-40.

[16] Hsiao JC, Chung CS, Chang W. Vaccinia virus envelope D8L protein binds to cell surface chondroitin sulfate and mediates the adsorption of intracellular mature virions to cells. J Virol 1999; 73: 8750-61.

[17] Hsiao JC, Chung CS, Chang W. Cell surface proteoglycans are necessary for A27L protein-mediated cell fusion: identification of the N-terminal region of $\mathrm{A} 27 \mathrm{~L}$ protein as the glycosaminoglycanbinding domain. J Virol 1998; 72: 8374-9.
[18] Lee HJ, Essani K, Smith GL. The genome sequence of Yaba-like disease virus, a Yatapoxvirus. Virology 2001; 281: 170-92.

[19] Cameron C, Hota-Mitchell S, Chen L, et al. The complete DNA sequence of myxoma virus. Virology 1999; 264: 298-318.

[20] Stanford MM, Barrett JW, Nazarian SH, Werden S, McFadden G. Oncolytic virotherapy synergism with signaling inhibitors: Rapamycin increases myxoma virus tropism for human tumor cells. J Virol 2007; 81: 1251-60.

[21] Testa JR, Bellacosa A. AKT plays a central role in tumorigenesis. Proc Natl Acad Sci USA 2001; 98: 10983-5.

[22] Jeong SJ, Pise-Masison CA, Radonovich MF, Park HU, Brady JN Activated AKT regulates NF-kappaB activation, p53 inhibition and cell survival in HTLV-1-transformed cells. Oncogene 2005; 24: 6719-28.

[23] Chen J, Somanath PR, Razorenova O, et al. Akt1 regulates pathological angiogenesis, vascular maturation and permeability in vivo. Nat Med 2005; 11: 1188-96.

[24] Aoki M, Batista O, Bellacosa A, Tsichlis P, Vogt PK. The akt kinase: molecular determinants of oncogenicity. Proc Natl Acad Sci USA 1998; 95: 14950-5.

[25] Werden SJ, McFadden G. The role of cell signaling in poxvirus tropism: the case of the M-T5 host range protein of myxoma virus. Biochim Biophys Acta 2008; 1784: 228-37.

[26] Lun X, Yang W, Alain T, et al. Myxoma virus is a novel oncolytic virus with significant antitumor activity against experimental human gliomas. Cancer Res 2005; 65: 9982-90.

[27] Lun XQ, Zhou $\mathrm{H}$, Alain $\mathrm{T}$, et al. Targeting human medulloblastoma: oncolytic virotherapy with myxoma virus is enhanced by rapamycin. Cancer Res 2007; 67: 8818-27.

[28] Barrett JW, Alston LR, Wang F, et al. Identification of host range mutants of myxoma virus with altered oncolytic potential in human glioma cells. J Neurovirol 2007; 13: 549-60.

[29] Wu Y, Lun X, Zhou H. Oncolytic efficacy of recombinant vesicular stomatitis virus and myxoma virus in experimental models of rhabdoid tumors. Clin Cancer Res 2008; 14: 1218-27.

[30] Woo Y, Kelly KJ, Stanford MM. Myxoma virus is oncolytic for human pancreatic adenocarcinoma cells. Ann Surg Oncol 2008; 15: 2329-35.

[31] Wang G, Barrett JW, Stanford M, et al. Infection of human cancer cells with myxoma virus requires Akt activation via interaction with a viral ankyrin-repeat host range factor. Proc Natl Acad Sci USA 2006; 103: 4640-5.

[32] Prag S, Adams JC. Molecular phylogeny of the kelch-repeat superfamily reveals an expansion of $\mathrm{BTB} / \mathrm{kelch}$ proteins in animals. BMC Bioinformatics 2003; 42: 1-20.

[33] Downie AW, España C. Comparison of Tanapox virus and Yabalike viruses causing epidemic disease in monkeys. J Hyg (Lond) 1972; 70: 23-32.

[34] $\mathrm{Hu} \mathrm{Y}$, Lee J, McCart JA, et al. Yaba-like disease virus: an alternative replicating poxvirus vector for cancer gene therapy. J Virol 2001; 75: 10300-8. 